\section{Starke Kombination von Thymian mit Efeu und Primel}

\begin{abstract}
— Um den Leidensdruck von Patienten mit akutem Erkältungshusten zu lindern und die Hustenanfälle effektiv zu reduzieren, eignet sich besonders das Phytotherapeutikum Bronchipret ${ }^{\oplus}$. Aufgrund der hervorragenden klinischen Studienlage wurde die Pflanzenextraktkombination ausschließlich diesen Präparates mit der Empfehlung „Stark“ in die Leitlinien der Deutschen Gesellschaft für Pneumologie und Beatmungsmedizin (DGP) aufgenommen. Auch in einer großen Praxisstudie mit Kindern wurde seine sehr gute Wirksamkeit und Verträglichkeit bestätigt.

Zwei klinische Studien belegen bei der Indikation akuter Erkältungshusten die Wirksamkeit und Verträglichkeit von Bronchipret ${ }^{\circledR}$. Das Phytotherapeutikum enthält Wirkstoffe aus Thymian und Efeu (Saft) bzw. Thymian und Primel (Filmtabletten). Beide Studien wurden randomisiert, placebokontrolliert und doppelblind durchgeführt. In die Studie aufgenommen wurden Patienten mit mindestens zehn Hustenanfällen täglich, einer seit maximal zwei Tagen bestehenden bronchialen Schleimproduktion mit erschwertem
\end{abstract}

\section{CED im Fokus}

— Um bei Kindern mit einer chronischentzündlichen Darmerkrankung (CED) ein normales Wachstum und eine regelrechte Pubertätsentwicklung zu gewährleisten, ist ein frühe Diagnose erforderlich. Noch aber ist die Latenz bis zur Diagnosestellung viel zu lang, bemängelte Dr. Martin Claßen aus Bremen beim Pädiater-Forum Gastroenterologie in München. Nicht nur bei blutigen Durchfällen dürfe an eine CED gedacht werden. Auch extraintestinale Symptome seien oft wegweisend, allen voran Gedeih- und Wachstumsstörungen.,Nicht selten wird eine CED deswegen als Anorexia nervosa fehldiagnostiziert." Und: Ist die Entzündung bei Morbus Crohn nicht im Kolon, sondern im oberen Gastointestinaltrakt lokalisiert, fehlt der Durchfall als typisches Symptom und die Koloskopie ist ohne Befund.

Die Behandlung einer CED sollte möglichst rasch eingeleitet werden. Zur Remissionsinduktion bei Morbus Crohn steht die Ernährungstherapie im Vordergrund. Bei leichter bis
Abhusten und einem Bronchitis-SymptomenScore von mindestens 5. Die Ergebnisse der Verumgruppe waren signifikant besser als unter Placebo. Mit Thymian-Efeu-Saft verringerte sich die Anzahl der Hustenanfälle um 68,7\% (Placebo: 47,6\%), mit Thymian-PrimelFilmtabletten um 67,1\% (Placebo: 51,3\%). Bei beiden Darreichungsformen konnte die Zahl der Hustenanfälle zwei Tage früher halbiert werden als unter Placebo.

Ergänzt wurden diese an Erwachsenen durchgeführten Studien von einer Anwendungsbeobachtung mit Kindern. Diese prospektive Untersuchung mit 1.234 Kindern und Jugendlichen im Alter von zwei bis 17 Jahren zeigte auch bei jungen Patienten die gute Wirksamkeit der Pflanzenextraktkombination. Die Hustenintensität verringerte sich schnell, nach nur vier Tagen war der Bronchitis-Symptomen-Score bereits um die Hälfte reduziert. Die Verträglichkeit wurde mit sehr gut bewertet.

Nach Informationen von Bionorica SE, Neumarkt

mittelgradiger Entzündung können auch 5-Aminosalizylate wie Mesalazin (z.B. Salofalk ${ }^{\circledR}$ Tabletten) versucht werden. Ihre Domäne sind allerdings in erster Linie Remissionsinduktion und -erhalt bei leichter bis mittelgradiger Colitis ulerosa. Eher kritisch bewertet Dr. Stefan Buderus aus Bonn den Einsatz von Probiotika. Hier lägen nur wenige positive Therapiestudien vor, die einen lediglich moderaten Effekt zeigten. Ist die entzündliche Aktivität mäßig bis schwer, sind für die Remissionsinduktion Steroide indiziert. Viele Kinder benötigen als effektive Erhaltungstherapie zusätzlich ein Immunsuppressivum wie Azathioprin (z.B. Azafalk ${ }^{\circledast}$ ). Initial eingesetzt, lassen sich die Remissionsraten einer Studie zufolge deutlich verbessern und Steroide einsparen. Buderus plädierte deshalb dafür, bei Colitis ulcerosa und M. Crohn die Kinder frühzeitig immunsuppressiv zu behandeln.

Nach Informationen der Falk Foundation e.V., Freiburg

\section{Prebiotika reduzieren} Allergierisiko

Für alle Mütter allergiegefährdeter Säuglinge, die nicht stillen können, hat Aptamil eine hypoallergene Säuglingsnahrung entwickelt, die das Allergierisiko langfristig signifikant reduziert. Eine Studie aus Mailand an 206 allergiegefährdeten Kindern von Moro $\mathrm{G}$ et al. beleget die gute Wirksamkeit: In der prospektiven, randomisierten Doppelblindstudie erhielten 40 Säuglinge in den ersten sechs Monaten HA-Nahrung mit einer patentierten Prebiotics-Mischung (0,8 g/100 ml scGOS/ IcFOS). Die 49 Kinder der Kontrollgruppe erhielten HA-Nahrung ohne Prebiotica. Im Beobachtungszeitraum von fünf Jahren litten $20 \%$ der Kinder in der PrebioticsGruppe unter atopischer Dermatitis. In der der Kontrollgruppe waren es $38,8 \%$. Auch in Bezug auf die Inzidenz bei Heuschnupfen gab es signifikante Unterschiede: So zeigten in der Prebiotica-Gruppe nur 2,5\% aller Kinder Symptome, in der Kontrollgruppe waren es hingegen $16,3 \%$. Bereits die 6-Monatsergebnisse hatten gezeigt, dass sich durch die Supplementierung der Nahrungen mit Prebiotika die Inzidenz von atopischer Dermatitis um rund $50 \%$ reduzieren ließ.

Nach Informationen von Milupa, Friedrichsdorf

Impfungen: Informationsbedarf bei den Eltern

Die Mehrheit der Eltern von Kindern im Alter von 0-13 Jahren folgt den Impfempfehlungen der Ständigen Impfkommission (STIKO), so das Ergebnis einer aktuellen Studie der Bundeszentrale für gesundheitliche Aufklärung (BZgA) mit über 3.000 Eltern. Doch vor allem bei Masern, Keuchhusten und Hepatitis B sei der Impfschutz noch immer lückenhaft. Laut BZgA-Studie möchten $98 \%$ der Eltern zum Thema Kinderimpfungen von ihrem Arzt informiert werden. Auch Informationsbroschüren und Faltblätter zu Impfungen sind bei vielen Eltern gefragt ( $81 \%)$. Besonders Eltern mit Migrationshintergrund äußern einen deutlichen Informationsbedarf. Daneben gewinnt das Internet gemäß Studien zunehmend an Bedeutung als Informationsmedium zum Impfen. Die BZgA stellt daher zusätzlich zu verschiedenen, auch fremdsprachigen Printmedien umfangreiche Informationen auf ihrem Internetportal www.impfen-info.de bereit.

Nach Informationen der BZgA, Köln 